\title{
NLRP3 Inflammasome: A Novel Therapeutic Target in Arthritis
}

\section{Enas Samir Nabih *}

Department of Medical Biochemistry, Faculty of Medicine, Ain Shams University, Cairo, Egypt

*Corresponding author: Enas Samir Nabih, Department of Medical Biochemistry, Faculty of Medicine, Ain Shams University, Cairo, PO 38, Egypt, Tel: +201223180464; E-mail: enassamer@hotmail.com

Received date: June 04, 2018; Accepted date: June 05, 2018; Published date: June 06, 2018

Copyright: $\odot 2018$ Nabih ES. This is an open-access article distributed under the terms of the Creative Commons Attribution License, which permits unrestricted use, distribution, and reproduction in any medium, provided the original author and source are credited.

\section{Editorial}

Arthritis is a term used to mean an inflammation of one or more of your joints. The main symptoms of arthritis are joint pain and stiffness, which typically worsen with age. Other symptoms may include redness, warmth, and swelling and decreased range of motion of the affected joints. The most common types of arthritis are osteoarthritis and rheumatoid arthritis [1].

The inflammasome is a multiprotein intracellular complex that plays an important role in inflammation. It also induces a lytic form of regulated cell death termed pyroptosis [2].

Pyroptosis has been reported in macrophages infected with Shigella flexneri and Salmonella enterica serovar Typhimurium bacterial pathogens [3-5]. This term is used since 2001 to distinguish this inflammatory form of caspase-regulated necrosis from accidental necrosis and apoptosis [6].

Pyroptosis induction requires two types of receptors that sense harmful signals which can be given off by invasive pathogens or by an injury to a tissue. These receptors are Nod-like receptors (NLRs) and Toll-like receptors [7]. The nucleotide binding domain and leucinerich repeat pyrin 3 domain (NLRP3 or cryopyrin) sensor protein, organizes the assembly of the best-characterized inflammasome, the NLRP3-inflammasome. NLRP3 is kept in an inactive state complexed with Heat Shock Protein 90 (HSP90) and suppressor of the G2 allele of skp1 (SGT1) in the cytoplasm. Upon detecting harmful signals, HSP90 and SGT1 are released from NLRP3 which then recruits apoptosisassociated speck-like protein containing a CARD (ASC) protein and caspase-1 to the inflammasome complex. ASC contains a caspase activation and recruitment domain (CARD) that binds and facilitates activation of pro-caspase-1 through CARD-CARD interactions. Activated caspase- 1 cleaves the precursors of interleukin (IL)- $1 \beta$ and IL-18 converting them into the mature, secreted inflammatory cytokines [8]. Furthermore, the inflammatory caspases 1, 4 and 5 can induce pyroptosis directly by cleaving gasdermin D (GSDMD) into a pore-forming amino-terminal domain (GSDMDN) and an inhibitory carboxy-terminal (GSDMDC) domain. GSDMDN then oligomerizes and inserts in the plasma membrane inducing rapid cell lysis [9-12].

There is emerging evidence for involvement of the NLRP3inflammasome/IL-1 $\beta$, IL18 axis in the inflammatory responses of arthritic disorders namely osteoarthritis [2,13], and rheumatoid arthritis [14]. The study of Sorge et al. [15] showed that active rheumatoid arthritis is associated with increasing NARP3 expression and IL- $1 \beta$ secretion in whole blood cells of mice upon stimulation via TLR3 and TLR4. In the same context, Choulaki et al. [14] revealed that IL- $1 \beta$ secretion seems to be predominately driven by caspase- 1 and caspase-8. They postulated that targeting NARP3 or downstream caspases may be of benefit in suppressing IL- $1 \beta$ production in rheumatoid arthritis.
In the recent study done by Zhao et al. [13], NLRP1 and NLRP3 inflammasomes expression was found to be high in human synovial samples obtained from knee osteoarthritis patients. When they isolated human fibroblast-like synoviocytes (FLSs) in vitro and stimulated them with lipopolysaccharide (LPS) and ATP, this resulted in cell pyroptosis. Interestingly, LPS+ATP-induced pyroptosis was attenuated by NLRP1 and NLRP3 siRNAs. Additionally, inhibition of NLRP1 and NLRP3 led to a remarkable reduction of pyroptosis-related cytokines.

In conclusion, NLRP3 inflammasome may have a potential role in the pathogenesis of arthritis. Targeting NLRP3 may represent a novel therapeutic target in arthritis.

\section{References}

1. Sofi F, Ahmad M, Bilal, Ashiq, Arif (2018) Efficacy of Intra-Articular Steroid Injection in Osteoarthritis Patients. J Arthritis 7: 269.

2. McAllister MJ, Chemaly M, Eakin AJ, Gibson DS, McGilligan VE (2018) NLRP3 as a potentially novel biomarker for the management of osteoarthritis. Osteoarthritis Cartilage 26: 612-619.

3. Chen Y, Smith MR, Thirumalai K, Zychlinsky A (1996) A bacterial invasin induces macrophage apoptosis by binding directly to ICE. EMBO J 15: 3853-3860.

4. Hersh D, Monack DM, Smith MR, Ghori N, Falkow S, et al. (1999) The Salmonella invasin SipB induces macrophage apoptosis by binding to caspase-1. Proc Natl Acad Sci USA 96: 2396-2401.

5. Monack DM, Raupach B, Hromockyj AE, Falkow S (1996) Salmonella typhimurium invasion induces apoptosis in infected macrophages. Proc Natl Acad Sci USA 93: 9833-9838.

6. Cookson BT, Brennan MA (2001) Pro-inflammatory programmed cell death. Trends Microbiol 9: 113-114.

7. Bortoluci KR, Medzhitov R (2010) "Control of infection by pyroptosis and autophagy: role of TLR and NLR Cell". Cell Mol Life Sci 67: 1643-1651.

8. Lamkanfi M, Dixit VM (2014) Mechanisms and functions of inflammasomes. Cell 57: 1013-1022.

9. Kayagaki N, Stowe IB, Lee BL, O'Rourke K, Anderson K, et al. (2015) Caspase-11 cleaves gasdermin $\mathrm{D}$ for non-canonical inflammasome signalling. Nature 526: 666-671.

10. Shi J, Zhao Y, Wang K, Shi X, Wang Y, et al. (2015) Cleavage of GSDMD by inflammatory caspases determines pyroptotic cell death. Nature 526 : 660-665.

11. Aglietti RA, Estevez A, Gupta A, Ramirez MG, Liu PS, et al. (2016) GsdmD p30 elicited by caspase-11 during pyroptosis forms pores in membranes. Proc Natl Acad Sci USA 113: 7858-7863.

12. Ding J, Wang K, Liu W, She Y, Sun Q, et al. (2016) Pore-forming activity and structural autoinhibition of the gasdermin family. Nature 535: 111-116.

13. Zhao LR, Xing RL, Wang PM, Zhang NS, Yin SJ, et al. (2018) NLRP1 and NLRP3 inflammasomes mediate LPS/ATP induced pyroptosis in knee osteoarthritis. Mol Med Rep 17: 5463-5469. 
Citation: Nabih ES (2018) NLRP3 Inflammasome: A Novel Therapeutic Target in Arthritis. J Arthritis 7: e118. doi:10.4172/2167-7921.1000e118

Page 2 of 2

14. Choulaki C, Papadaki G, Repa A, Kampouraki E, Kambas K, et al. (2015) Enhanced activity of NLRP3 inflammasome in peripheral blood cells of patients with active rheumatoid arthritis. Arthritis Res Ther 17: 257.

15. Sorge, RE, LaCroix-Fralish ML, Tuttle AH, Sotocinal SG, Austin JS, et al. (2011) Spinal cord Toll-like receptor 4 mediates inflammatory and neuropathic hypersensitivity in male but not female mice. J Neurosci冈31: 15450-15454. 